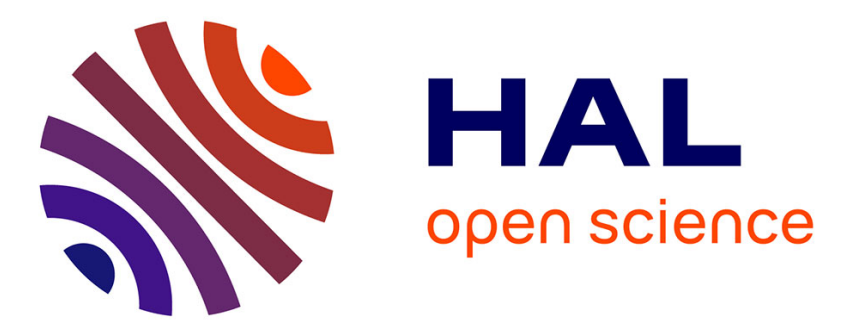

\title{
La station antique de Lero à l'île Sainte-Marguerite (Alpes-Maritimes)
}

Jules Formigé

\section{To cite this version:}

Jules Formigé. La station antique de Lero à l'île Sainte-Marguerite (Alpes-Maritimes). Gallia - Fouilles et monuments archéologiques en France métropolitaine, 1947, 5 (1), pp.146-155. 10.3406/galia.1947.2021 . hal-01921033

\section{HAL Id: hal-01921033 \\ https://hal.science/hal-01921033}

Submitted on 6 Feb 2020

HAL is a multi-disciplinary open access archive for the deposit and dissemination of scientific research documents, whether they are published or not. The documents may come from teaching and research institutions in France or abroad, or from public or private research centers.
L'archive ouverte pluridisciplinaire HAL, est destinée au dépôt et à la diffusion de documents scientifiques de niveau recherche, publiés ou non, émanant des établissements d'enseignement et de recherche français ou étrangers, des laboratoires publics ou privés.

\section{(이) $\$$}

Distributed under a Creative Commons Attribution - NonCommercial - NoDerivatives $\mid 4.0$ 
rugueuse, assez tendre. Sur une grande anse ronde.

18. SE ?. Poterie rougeâtre fine, assez dure, paillettes de mica doré.

19. SOTIC. Poterie rougeâtre, jaunâtre en surface, dure. Sur un col.

20. ? VOLTEILI. Mème poterie que 18.

21. S (?) C (?) IA. Poterie, fine, dure.

22. B (ou P) N. Poterie rougeâtre assez tendre. Mêmes paillettes que 10 et 14. Sur la base d'une anse plate.

23. ? AGN. Même poterie que 18 et 20. Sur un col.

24. $\mathrm{X}$, dans un cachet rond. Cette marque est peut-ètre grecque (cf. fig. 2, $n^{0}$ 6). Argile claire, assez mal épurée, parsemée de fines paillettes de mica doré, bien cuite, rosée à la cassure, jaune clair à l'intérieur. Sur un petit fragment de col.

25. A, tracé avant cuisson sur la panse d'une amphore. Argile rougeâtre rugueuse et bien cuite.

26. E (?). Légèrement gravé après cuisson un peu au-dessous d'une épaule bien marquée, à angle droit. Même argile que 25. Ce tesson porte encore à l'intérieur quelques plaques d'un enduit noirâtre et craquelé (poix ou tartre).

27 . $R$ (?) rétrograde tracé avant cuisson sur la pointe d'une amphore (forme 7 de Dressel). Parois minces, terre très dure et rugueuse, brunâtre à la cassure, jaune clair à l'intérieur.

Les tessons de ces amphores se rencontrent dans les niveaux $\mathrm{V}$ et VI (en gros, fin II $^{\mathrm{e}}$ siècle av. J.-C., II $^{\mathrm{e}}$ après).

Odette et Jean Taffanel.

\section{la Station antigue de LERo \\ A L'ILE SAINTE-MARguerite \\ (ALPES-MARITIMES)}

Les itinéraires maritimes antiques indiquent aux iles de Lérins, en face de Cannes, la station de Lero (fig. 1). Une inscription conservée au couvent de Saint-Honorat y mentionne un collège des "utriculaires "1. Dans le cloitre de ce couvent, on a rassemblé de nombreux fragments romains : statuaire, éléments d'architecture, sarcophages et inscriptions. A la base de la face orientale du donjon on voit également quelques lettres d'une grande inscription romaine.

On pourrait cependant se demander si ces fragments n'ont pas été apportés d'ailleurs. Mais une grande statue de
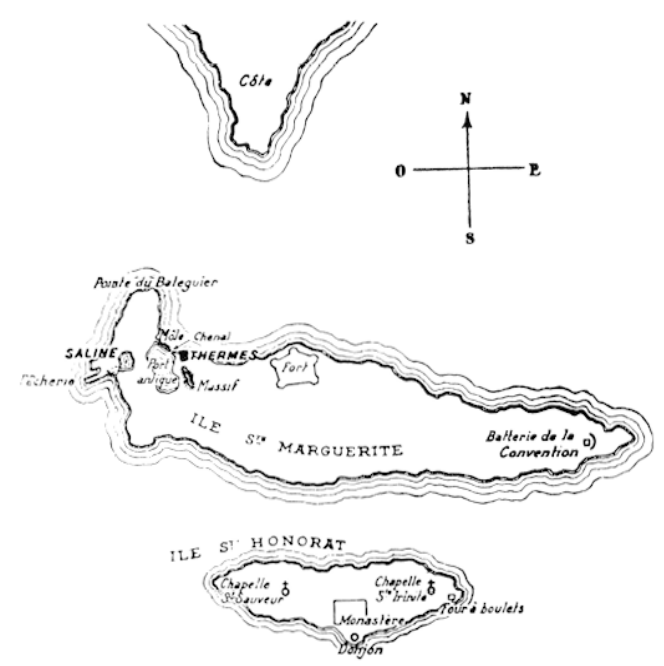

Fig. 1. - L'ile Sainte-Marguerite et l'ile Saint-Honorat (iles de Lérins).

Priape, découverte en 1937 par des moines qui cultivaient une vigne, prouve que l'lle Saint-Honorat a bien été habitée par les Romains. En effet, les religieux qui occupent cette ile depuis le ve siècle n'y auraient pas apporté une statue de ce type.

Ces données étaient les seules que nous possédions jusqu'en 1937. Elles ne constituaient que des souvenirs de la station

(1) Trouvée dans l'ile de Lérins, aujourd'hui au couvent de Saint-Honorat. CIL, XII, 187 : Collegis utricular(iorum), C. Iulius Calullinus don(o) pos (uit). 
disparue, dont l'emplacement lui-même était oublié.

Pour le retrouver, je ne pouvais donc pas partir de renseignements matériels ; mais en revanche les lois de la nature et du climat local n'ayant pas changé, il était évident que, pour choisir leur emplacement, les constructeurs antiques avaient dû en tenir compte. Posé ainsi, le problème cessait d'être de l'archéologie et ne relevait plus que du bon sens : y avait-il plusieurs endroits pro-

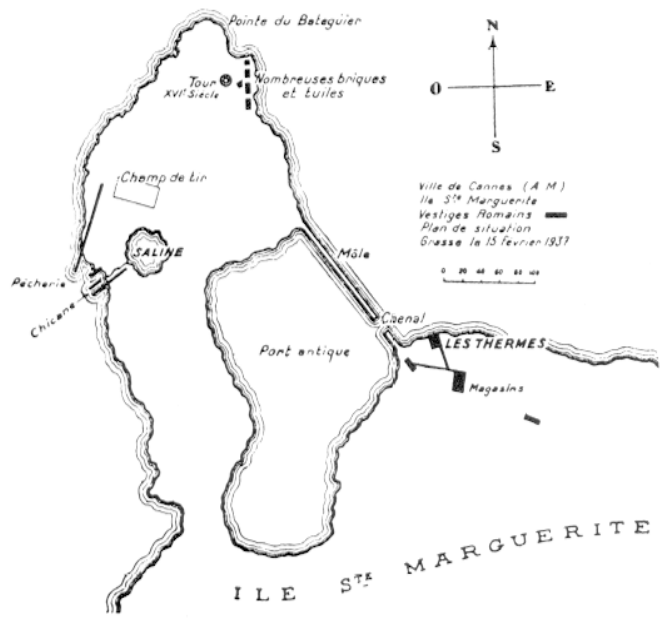

Fig. 2. - L'île Sainte-Marguerite.

Le port antique et les vestiges romains.

pres à établir un port et, s'il y en avait plusieurs, quel serait le meilleur?

Ayant fait le tour de l'ile SaintHonorat en barque, je constatai qu'elle était entourée de tous côtés de récifs fort dangereux, que des courants violents rendaient encore plus redoutables. Malgré un appontement en béton créé récemment au sud de cette ile, dans le détroit qui la sépare de sa voisine, il y a des jours où il est impossible d'aborder.

J'entrepris alors de faire le tour de l'ile Sainte-Marguerite et je constatai qu'il y avait un endroit et un seul où un port était possible. Il est situé au nord- ouest de l'ile (fig. 2). On y trouve une grève, dans une anse abritée de tous les vents, précisément à l'endroit où l'on a établi les appontements modernes qui servent aux pêcheurs et aux touristes. Un simple coup d'œil me fit voir, parmi les galets du rivage, de nombreux fragments de poteries communes, de dolia, d'amphores grecques et romaines et de tuiles à rebords : il est surprenant, vu les innombrables personnes qui sont passées en cet endroit, que nul ne les ait remarqués.

Au delà du rivage, se trouve un étang aux eaux dormantes assez vastes. Étaitce un élément de l'ancien port ensablé ? Un simple coup de filet de pêcheurs opéré sur ma demande dans cet étang ramena une quantité de fragments d'amphores grecques et romaines. Enfin un sondage opéré sur la grève entre la mer et l'étang rencontra le Môle.

L'emplacement de la station antique était ainsi retrouvé avec précision.

Les raisons du choix de cet emplacement sont nombreuses. Tout d'abord la situation de cette ile, entre le cap d'Antibes qui l'abrite du vent d'est et l'Esterel qui coupe le mistral, en fait le point où se produisent les sautes de ces vents contraires et où une escale s'impose aux voiliers. Quant au grand vent du large, l'anse en est protégée par l'ille ellemême.

Établie dans cette ile au lieu d'être sur la côte, la station était plus facile à défendre. Et elle n'était distante de cette côte que de moins d'un kilomètre, ce qui permettait d'y atteindre facilement. Les utriculaires se trouvaient tout spécialement qualifiés pour ce trafic, grâce à leurs radeaux d'outres au faible tirant d'eau, pouvant porter de grosses charges et ne redoutant pas le choc des écueils.

D'ailleurs on sait que, jusqu'à l'époque d'Auguste, la voie côtière n'existait pas 
encore, et qu'il ne se trouvait à son futur emplacement qu'une piste historique peu sûre. Presque tout le trafic se faisait donc par mer, entre les comptoirs où s'opéraient les échanges des productions du pays contre les marchandises importées. La route côtière fut d'ailleurs bien négligée par la suite, puisque l'enquête ordonnée par Napoléon Ier et publiée récemment nous apprend qu'au début du $x_{1 x}{ }^{e}$ siècle il n'y avait plus aucune route carrossable.

La valeur de cette station est attestée dans l'histoire par tous les assauts succes- fortins espagnols et la forteresse bâtie par Vauban où fut emprisonné le célèbre Masque de Fer ; et à l'extrême pointe nord-ouest, la pointe de Batéguier, une tour ronde commandant les rivages de l'ouest et du nord. Pour ces constructions comme pour le couvent de SaintHonorat, les ruines antiques servirent de carrière de pierre.

Ayant repéré la station, je fis un examen rapide de ses environs immédiats. Au bord de la mer, au sud, sous une épaisse couche d'algues, je reconnus l'angle d'une salle romaine avec hypo-

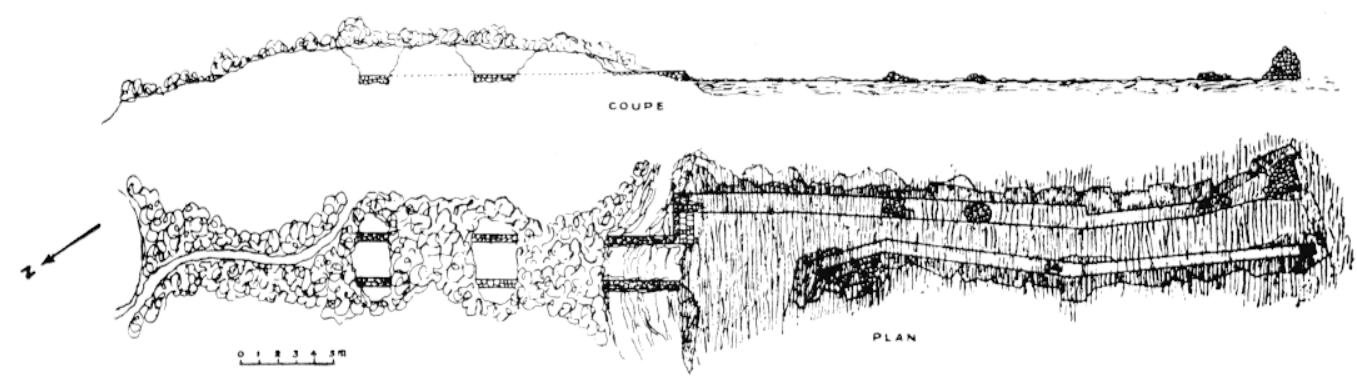

Fig. 3. - L'île Sainte-Marguerite. La chicane de Portègue, coupe et plan.

sifs qu'elle subit et par les maitres nombreux qui se la disputèrent. La divinité locale Lero remonte sans aucun doute aux pirates ligures. Les Grecs de Marseille puis les Romains pratiquèrent certainement cette escale. Puis ce furent les Barbares, Normands, Maures et autres, puis les Espagnols au Xvi ${ }^{\theta}$ siècle. C'est même là qu'en 1543 la flotte de Soliman II-le-Grand séjourna, lorsqu'elle fit sa jonction avec la flotte française pour lutter contre Charles-Quint. L'endroit s'appelait alors le Port des Galères. En 1544 Jérôme Maurand, prêtre d'Antibes, parle des "très belles antiquités" qui se dressaient encore sur le rivage et qu'il a vues. Elles n'étaient donc pas encore détruites. Mais on édifia alors de nouvelles constructions : d'abord les caustes. Sous la tour du nord-ouest qui date peut-être du xvie siècle, je remarquai une construction beaucoup plus ancienne et plus large, circulaire comme elle, qui pourrait être la base d'un ancien phare.

Au delà de cette tour, le long du rivage en direction nord-sud, je notai un immense mur difficile à dater. Puis, une série de murs romains en chicanes (fig. 3) avec lits de briques, qui s'enfoncent dans la mer peu profonde en cet endroit, et qui constituaient peut-être une pêcherie ou une réserve (fig. 4). Un canal s'en détache et pénètre dans les terres. Il semble se diriger vers un bas-fond dont le radier rocheux a pu être utilisé pour une ancienne saline. Enfin, à l'est du port, je pus voir de nombreux restes de 
murs à fleur de sol ou noyés dans les massifs de lentisques.

Il est aussi à noter que les bâtiments des Eaux et Forêts sont orientés comme les murs antiques et vraisemblablement établis sur eux.

L'ensemble mesurait au moins $700 \mathrm{~m}$. de l'est à l'ouest. le mur du môle, épais de $1 \mathrm{~m}$., sur une longueur de plus de $170 \mathrm{~m}$., renforcé du côté de la mer par des contreforts espacés de $5^{\mathrm{m}}, 50$. Je n'ai pu constater dans cette première recherche si un second mur doublait le premier, mais j'ai retrouvé la passe d'entrée qui n'est qu'ensablée.

Les sondages furent continués par la

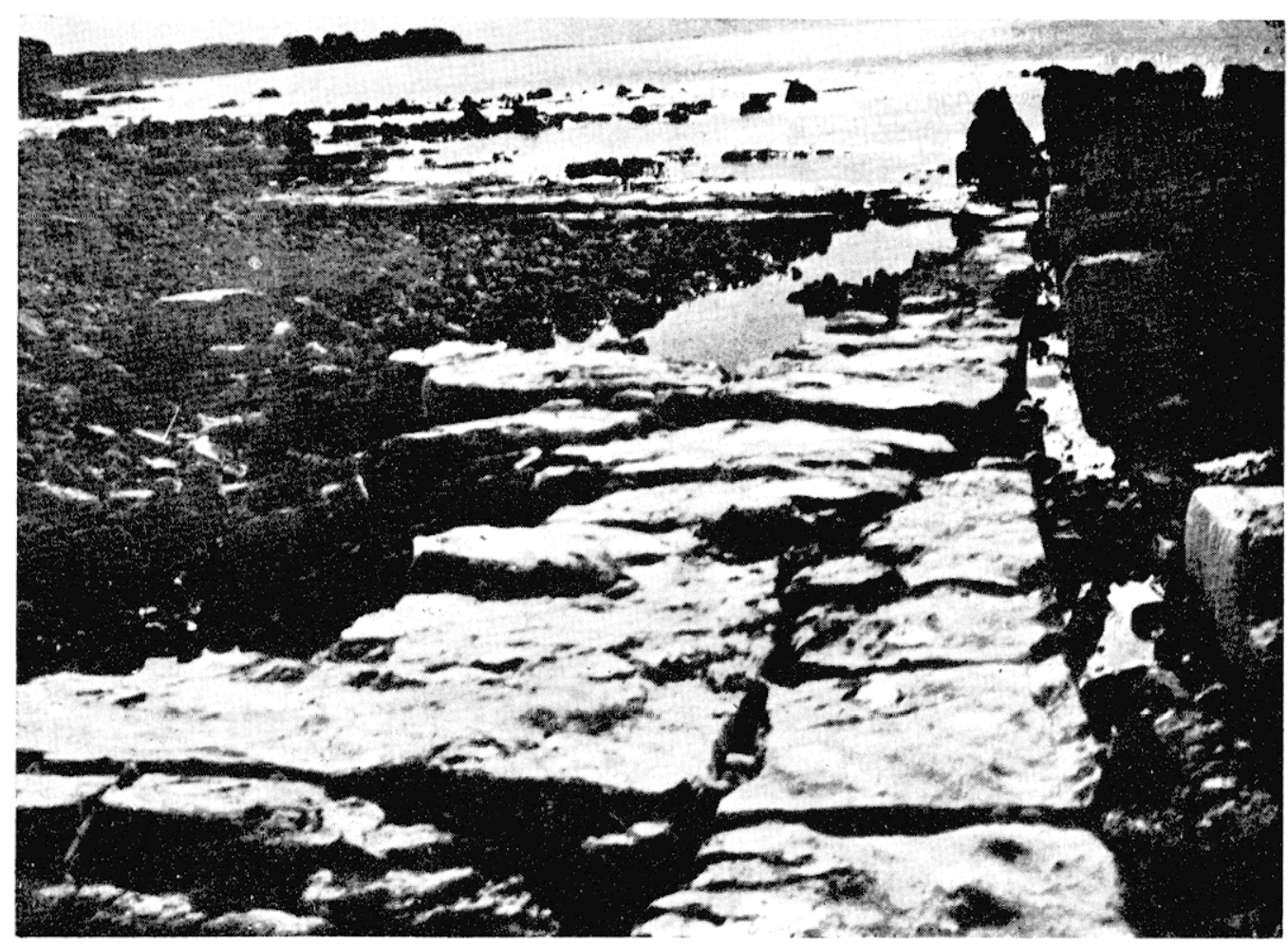

Fig. 4. - L'ile Sainte-Marguerite. Vue d'ensemble de la pêcherie romaine.

Ces constatations étant faites, il devenait facile d'entreprendre des sondages pour les amplifier. Je pus les commencer, avec l'aide de M. L. Le Bel, architecte des Monuments historiques des AlpesMaritimes et de M. Dor de la Souchère, conservateur du Musée d'Antibes, qui rivalisèrent de dévouement et d'ardeur dans cette entreprise.

Tout d'abord une série de sondages entre la mer et l'étang permit de repérer salle à hypocaustes A située à l'extrémité du môle, et qui appartient à des thermes (fig. 5). Des thermes situés au voisinage même de la mer ne sont pas sans exemple : à Olbia-Pomponiana près d'Hyères on en voit un établissement qui s'avance dans les flots. Et à Fréjus, au bord du port, existaient de très vastes thermes dont il nous reste la "porte dorée " et dont les fouilles de Texier ont révélé d'autres éléments. 
Malheureusement la construction des fortifications de Sainte-Marguerite et du couvent de Saint-Honorat, comme je l'ai dit plus haut, a fait raser les murs romains qu'on a exploités comme carrière, et cela d'autant plus qu'il n'existe pas de bonne pierre dans l'ile et qu'il est fort onéreux d'en amener de la côte.
Tous ces murs sont en petit appareil et les intérieurs conservent en partie leurs enduits.

Dans les déblais on a recueilli une quantité considérable de fragments de tuiles et de jarres brisées, de poteries campaniennes noires lustrées, de poteries samiennes, beaucoup de grands clous en

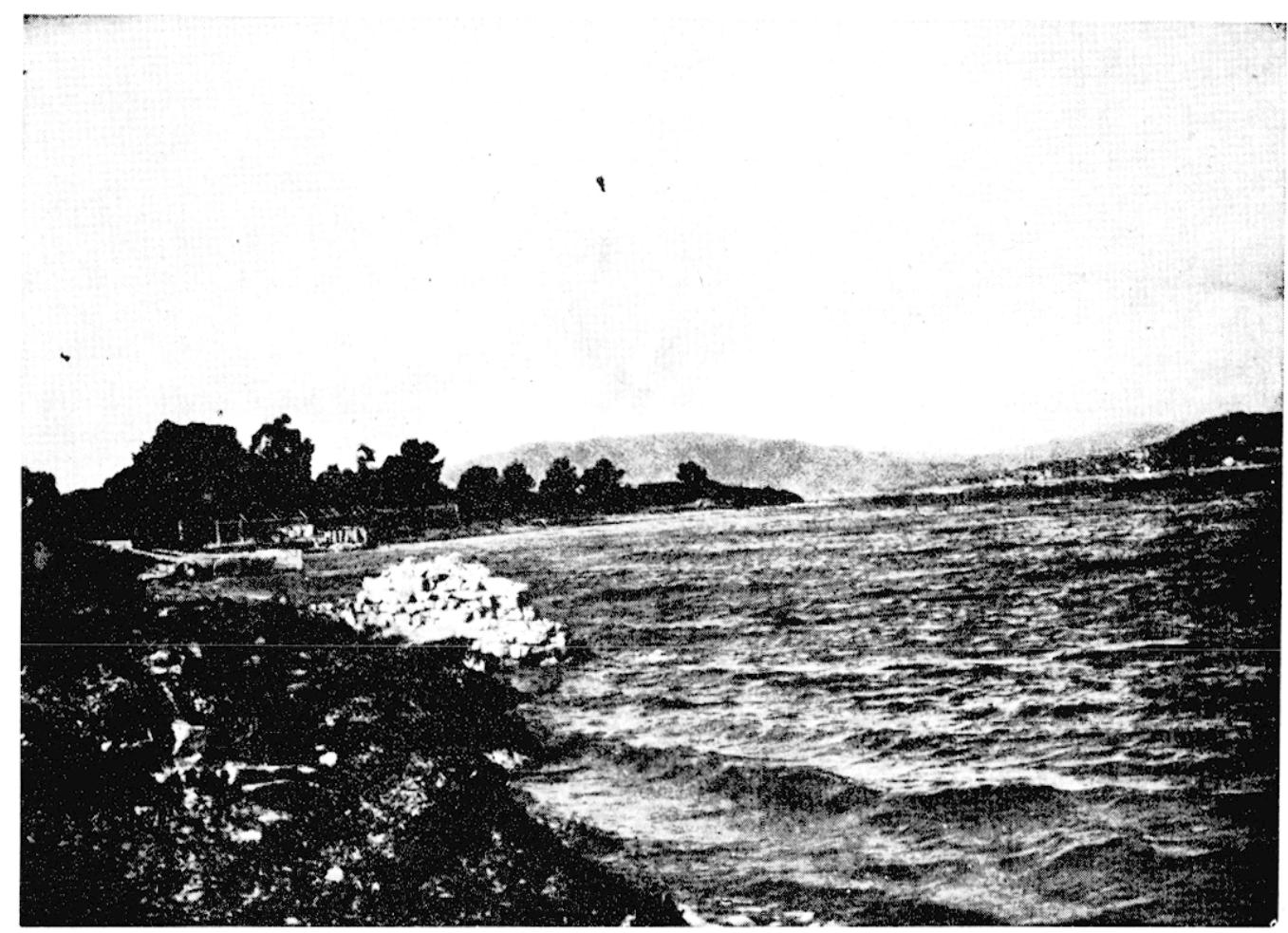

Fig. 5. - L'ile Sainte-Marguerite. Ruines des thermes antiques.

Huit salles ont été dégagées, A, B, C, $\mathrm{D}, \mathrm{E}, \mathrm{F}, \mathrm{G}, \mathrm{H}$. Les dimensions extrêmes de ce premier groupe de constructions atteignent $32 \mathrm{~m}$. sur 41. On y voit d'abord une salle sur hypocaustes $A$ avec l'entrée de son chauffage, salle malheureusement rongée par la mer. Il est difficile de préciser la destination de chacune de ces salles. Cependant on reconnait la fondation d'un portique $\mathrm{F}$ large de $2 \mathrm{~m}$. et long de 27. fer et un morceau d'inscription sur une plaque de marbre blanc mesurant $0^{\mathrm{m}}, 09$ de longueur, $0^{\mathrm{m}}, 05$ de hauteur et $0^{\mathrm{m}}, 02$ d'épaisseur. Les lettres sont larges, profondes, d'un bon tracé, bien gravées avec des empattements bien marqués. Elles sont de très bonne époque. J'ai cru lire :
A R A $[\mathrm{m}]$
[f] E C I T

$\mathrm{Au}$ sud de ces constructions s'étend 
un grand mur, de l'est à l'ouest, de $18 \mathrm{~m}$. de long, et qui semble s'y rattacher. Plus au sud encore, apparaissent d'autres murs jusqu'à la maison forestière où se voit un seuil en marbre blanc de $1^{\mathrm{m}}, 58$ sur $0^{\mathrm{m}}, 35$ et $0^{\mathrm{m}}, 25$ indiquant une construction plus luxueuse (fig. 6).

Une deuxième campagne de fouilles a permis de compléter les fouilles précédentes au sud des thermes. Elle a porté sur un périmètre qui mesure $26 \mathrm{~m}$. du nord au sud et $15 \mathrm{~m}$. de l'ouest à l'est. De plus, au nord un vaste mur qui s'en détache vers l'ouest a été suivi sur $42 \mathrm{~m}$. Il se dirige vers le môle.

Il faut remarquer que ce deuxième groupe de constructions est orienté aux points cardinaux alors que les thermes ne le sont pas. La raison n'en est pas connue. Il est à signaler que le mur nord est à contreforts, comme le môle, et sans doute de même époque.

Ce mur nord a été dégagé en premier. Il atteint à son point le plus haut $2^{\mathrm{m}}, \mathbf{4 0}$ au-dessus du sol romain. Sa masse est en blocage; ses parements en petit appareil de moellons soignés sur des joints saillants et tirés au fer. Il mesure $0^{\mathrm{m}}, 50$ d'épaisseur. Il s'appuie à l'angle nord-est sur une pile de $1^{\mathrm{m}}, 20$ de côté d'où se poursuit vers l'est un mur de $0^{\mathrm{m}}, 45$. A $0^{\mathrm{m}}, 75$ de cette pile d'angle un autre mur de $0^{\mathrm{m}}, 45$ se détache vers le nord. Dans son ensemble ce mur nord constitue la façade des constructions décrites ci-après. Il atteint $39 \mathrm{~m}, 90$ de long, avec six piles intermédiaires saillantes de $0^{\mathrm{m}}, 60$, larges de $0^{\mathrm{m}}, 92$ et dont l'écartement moyen est de $4^{\mathrm{m}}, 40$ sauf entre la cinquième et la sixième où cet écartement atteint $7 \mathrm{~m}, 20$.

A $3^{\mathrm{m}}, 87$ de la quatrième pile, un mur de $0^{\mathrm{m}}, 48$ vient se raccorder de biais avec ce mur nord, écornant l'angle nord-est du cinquième pilier. Ce mur, beaucoup moins bien fait et vraisemblablement moins ancien, forme le raccord avec les constructions portuaires décrites plus haut (thermes et annexes) qui seraient donc moins anciennes.

La travée entre la cinquième et la sixième pile, plus large que les autres, porte la marque d'une réfection très grossière. On ne peut donc deviner son état primitif, ni la raison de sa plus grande largeur, porte ou autre. Toutefois, dans cette partie, on voit un égout de $0^{\mathrm{m}}, 33$ de large et $0^{\mathrm{m}}, 48$ de profondeur, dont le radier est en tuiles à rebords, la couverture en lauzes vertes, et dont la pente s'abaisse vers le nord-ouest.

Enfin le massif correspondant à une pile d'angle se rattache à angle presque droit avec un mur sans parements long de $3^{\mathrm{m}}, 25$ se raccordant lui-même sous un angle de plus de 90 degrés avec le mur du môle en direction sud-estnord-ouest.

Passons maintenant au mur est qui est de mêmes dimensions et de même construction que le mur nord mais sans piles. Au bout de $12 \mathrm{~m}$. de longueur, sa fondation, assez grossière, remonte. A $13^{\mathrm{m}}, 72$ de la pile d'angle, on rencontre un mur de refend parallèle au mur nord qui délimite un premier rectangle mesurant $13^{\mathrm{m}}, 72$ sur $8^{\mathrm{m}}, 93$ dont le sol romain apparait bien de niveau.

Au delà de ce mur de refend, le mur est continue, mais en blocage et sans parements. Comme le sol des salles se relève en cet endroit, on peut penser que ce mur grossier n'est en réalité qu'une fondation.

Au sud du mur est, les constructions sont à peu près carrées et divisées en quatre salles sensiblement égales : $\mathrm{N}, 0$, $P, Q$, dont le sol est, comme je viens de le dire, à un niveau plus élevé que celui de la salle au nord du refend.

La première, $\mathrm{O}$, s'ouvre à l'est par un seuil en grès, gris violet, de $2^{\mathrm{m}}, 89$ de 


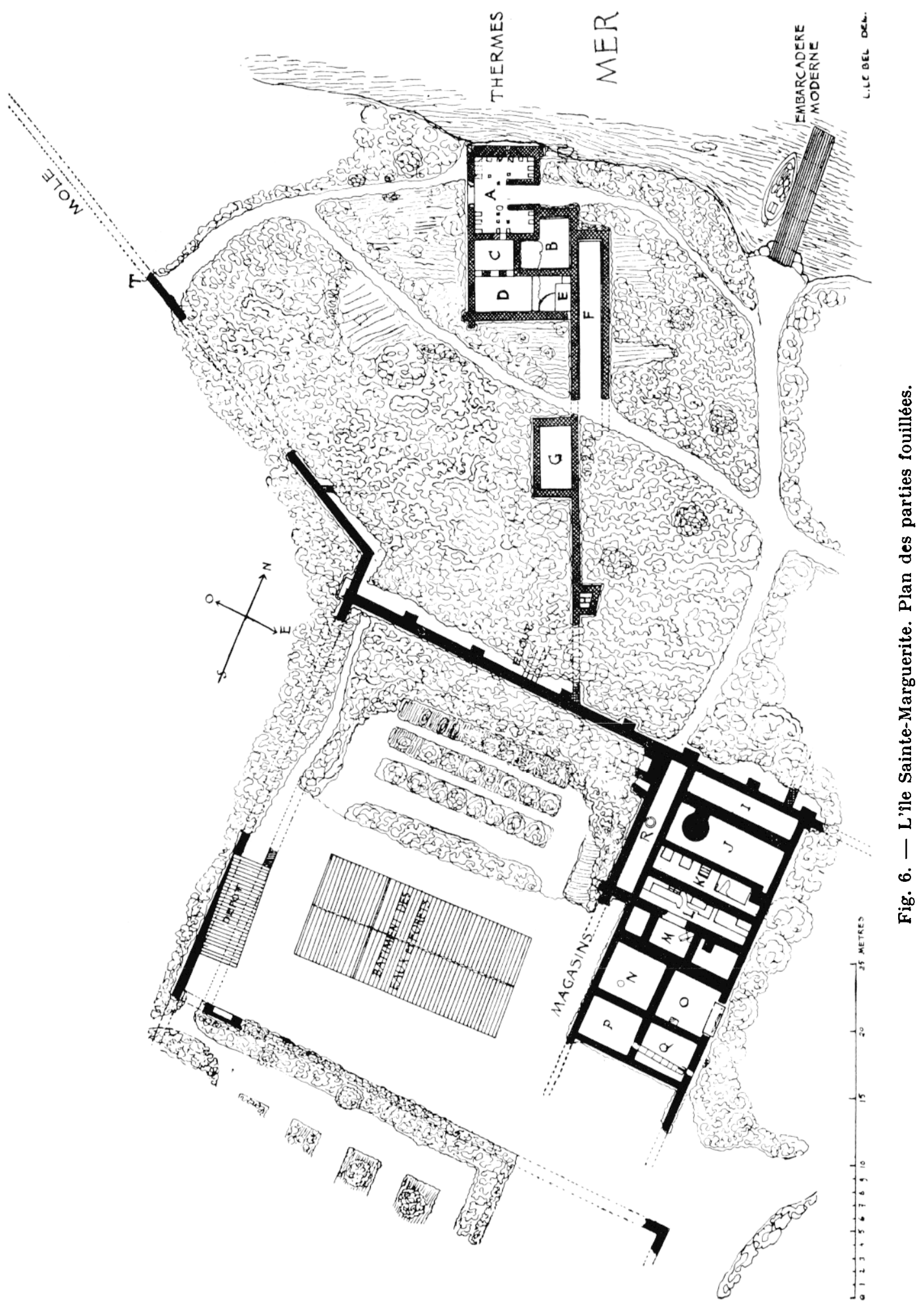


longueur, entaillé d'une rainure profonde servant, suivant l'usage, à engager la fermeture. Ce seuil est très usé du côté de l'intérieur et en revanche presque intact du côté de l'extérieur. Comme la rainure des volets montre que c'est bien un seuil, il faut supposer que le dallage extérieur était de niveau avec le seuil et que le dallage intérieur était légèrement en contrebas. Cette salle 0 mesure, du sud au nord, $4^{\mathrm{m}}, 07$. Un nouveau mur de refend la sépare de la salle suivante $Q$ qui mesure $3^{\mathrm{m}}, 20$ du sud au nord et dont l'ouverture vers l'est occupe toute cette largeur. Mais le seuil a disparu et il n'est resté que son emplacement sur un épais lit de chaux.

Le mur est atteint ainsi dans sa partie dégagée $(25 \mathrm{~m}$. de long). Il se continue ensuite vers le sud.

Nous en arrivons alors au mur ouest placé à $9 \mathrm{~m}, 12$ du mur est à la hauteur de la troisième pile du mur nord qui mesure comme lui $0^{\mathrm{m}}, 45$ d'épaisseur et présente la même construction. Sur sa face ouest s'allonge sur une longueur de $10^{\mathrm{m}}, 70$ une construction $R$ large de $1 \mathrm{~m}, 50$ dont le sol, en forte pente vers l'ouest, est à $1^{\mathrm{m}}, 30$ plus haut que celui des salles I, J, K, L, placées à l'est. Ce sol est garni d'un béton de chaux et brique pilée et le pied des murs est renforcé d'un quart de cylindre de $0^{\mathrm{m}}, 18$ en même matière. Les murs sont enduits de même. C'est très probablement une citerne.

Les fouilles y ont mis au jour un bloc de grès violacé circulaire, haut de $0^{\mathrm{m}}, 42$, atteignant $0^{\mathrm{m}}, 50$ de diamètre et qui, selon toute vraisemblance, est une base de colonne à deux tores séparés par un cavet, placée là sur une tuile à une fin qu'on ne peut deviner.

A l'angle nord-ouest de cette citerne supposée apparaissent des bases de construction d'un autre type avec mélange de moellons et de briques. Mais elles n'ont pas encore été dégagées.

$\mathrm{Au}$ sud de la citerne, le mur ouest continue. Comme pour le mur est il prend la forme d'une fondation, ce qui confirme que le sol se relevait en ce point.

J'en arrive à la description des murs de refend.

L'ensemble du quadrilatère dégagé par les fouilles est divisé, comme je l'ai dit plus haut, par un mur de refend à $13^{\mathrm{m}}, 72 \mathrm{du}$ mur nord. Dans cette première partie, la largeur entre le mur est et le mur ouest est de $9 \mathrm{~m}, 12$. Au sud de ce refend, l'espace libre mesure du nord au sud $7 \mathrm{~m}, 79$ et de l'est à l'ouest $8^{\mathrm{m}}, 90$. Mais, comme on est au niveau des fondations qui forment empattement, cette largeur devait être au-dessus de ces fondations de $9 \mathrm{~m}, 12$ également.

La première division en effet a son sol sensiblement plus bas que celui de la seconde : la différence est de $0^{\mathrm{m}}, 90$ environ.

Cette première division est recoupée par quatre murs de refend, tous parallèles au mur nord.

Le premier, à $1^{\mathrm{m}}, 97$ du mur nord, épais de $0^{\mathrm{m}}, 90$, n'est conservé que dans sa partie occidentale. Contre sa paroi sud s'adosse dans la salle $\mathfrak{J}$ une masse de moellons circulaire de $2 \mathrm{~m}$. de diamètre dont le dessus est enduit au mortier. Contre sa paroi nord se trouve la salle I.

Le deuxième à $3^{\mathrm{m}}, 03$ du précédent va du mur ouest au mur est sans être lié avec eux aux points où il les atteint. Il a $0^{\mathrm{m}}, 65$ d'épaisseur et est aussi en blocage peu soigné. Adossés à sa paroi sud dans la salle $\mathrm{K}$, sont cinq socles de $0^{\mathrm{m}}, 92$ de saillie et de $0^{\mathrm{m}}, 89$ de largeur moyenne. L'un d'eux est plus important; $2^{\mathrm{m}}, 38$ de long et $1 \mathrm{~m}$. de saillie. Celui du milieu porte encore un bloc de grès avec trois ressauts qui sont peut-être des marches. 
Le troisième mur de refend, épais de $0^{\mathrm{m}}, 80$, est à $2 \mathrm{~m}$. environ du précédent. Des empattements confus s'adossent dans la salle L à sa paroi sud et on $\mathrm{y}$ trouve également des gros blocs de grès violacé entaillés en forme de marches.

Le quatrième mur de refend se compose en réalité de deux tronçons affrontés. Deux murettes perpendiculaires, larges de $0^{\circ} \mathrm{m}, 55$, divisent l'espace en trois réduits $M$, vraisemblablement trois caves, vu le nombre des jarres qu'on y a retrouvées. Les largeurs de ces caves, de l'est à l'ouest sont respectivement $3^{\mathrm{m}}, 45,3^{\mathrm{m}}, 25$, $1^{\mathrm{m}}, 41$ sur une longueur moyenne nordsud de $2^{\mathrm{m}}, 70$ env.

Nous arrivons ainsi au cinquième mur qui est le grand refend signalé plus haut, à $13^{\mathrm{m}}, 72$ du mur nord.

Au sud de ce mur où la fouille s'arrête, on voit le départ d'un mur de $0^{\mathrm{m}}, 65$ perpendiculaire qui divise la largeur générale en deux salles nord-ouest à peu près égales. La salle orientale 0 s'ouvre dans le mur est par un vaste seuil large de $3 \mathrm{~m}$., en grès. A la suite de ces deux salles deux autres semblables $P, Q$, paraissent, divisant ainsi en croix l'espace situé au sud du cinquième refend. Mais comme le sol remonte de plus en plus on n'a retrouvé que l'amorce des fondations.

Il est impossible, vu l'état actuel des fouilles qui sont inachevées et vu aussi la ruine presque complète des constructions, d'en deviner la destination. Toutefois on a l'impression qu'il s'agit de locaux utilitaires et non d'habitations et que tous ces massifs, divers de forme et de grosseur, servaient de support à une machinerie.

Ces découvertes ne doivent être considérées que comme le début d'une fouille beaucoup plus vaste, dont il est impossible de prévoir les résultats. J'ai dit plus haut que j'avais repéré des cons- tructions antiques sur $700 \mathrm{~m}$. de long et les espaces fouillés ne mesurent dans le même sens que $32 \mathrm{~m}$. et $13 \mathrm{~m}$. respectivement. Cela suffit pour montrer ce qu'il reste \&े faire.

\section{Objets trouvés pendant la deurieme campagne des jouilles}

Les trouvailles sont peu nombreuses et sans valeur. Les locaux étaient évidemment pauvres.

Céramique. - Tuiles plates à rebords. Tuiles de recouvrement.

Petites briques carrées.

Grandes briques rectangulaires.

Briques circulaires pour les colonnes. Jarres du type I (Dressel, CIL, XV, pl. II) datant du dernier siècle de la République.

Les tuiles à rebords sont employées à des usages très variés en dehors des couvertures : piliers d'hypocaustes, radiers de canalisation, etc.

Pierre. - Une base de colonne à profil d'un seul tore dit toscan. Sa plinthe a $0^{\mathrm{m}}, 60$ de diamètre et la circonférence de la colonne est de $1^{\mathrm{m}}, 40$.

\section{Conclusion}

Telles sont actuellement nos connaissances sur les restes de la station navale de I.ero.

M. de la Souchère s'est demandé si ces installations portuaires ne s'accompagnaient pas également d'installations de pêche, de pisciculture, et de traitement des poissons. L'étang aurait servi de vivier, les murs pénétrant dans la mer à l'ouest de pêcheries, et l'adduction d'eau de mer dans la saline de récolte de sel pour le poisson. Cette hypothèse ingénieuse n'est pas à écarter car nous savons que les Romains, grands 
amateurs de poissons et de coquillages, avaient $d e$ nombreuses installations de ce genre au bord de la mer, soit artificielles, soil naturelles et aménagées.

\section{Jules Formigi: \\ FOUILLES A MARSEILLE DANS LE \\ QUARTIER DU VIEUX PORT \\ (PREMIERS SONDAGES)}

Au cours de l'occupation de Marseille, l'armée allemande a procédé à la démolition du vieux quartier étagé sur la rive septentrionale du port, entre la mer et la courbe de niveau suivie par la rue Caisserie. Le déblaiement des décombres, entrepris peu après par la municipalité, offrait une occasion unique, avant la reconstruclion prochaine, pour explorer le sol de Marseille à l'emplacement de la cité antique. Des sondages archéologiques ont été effectués ${ }^{1}$ de juin à octobre 1945 ; il s'agissait d'essayer de définir la limite nord du Lacydon et de rechercher, sur ce bord, des vestiges de la colonie grecque.

Trois tranchées ont été ouvertes (fig. 1).

Premier sondage. A l'endroit où l'ancienne rue "Bernard

(1) Cette mission m'a été confiée par lettre ministérielle du 23 mars 1945.

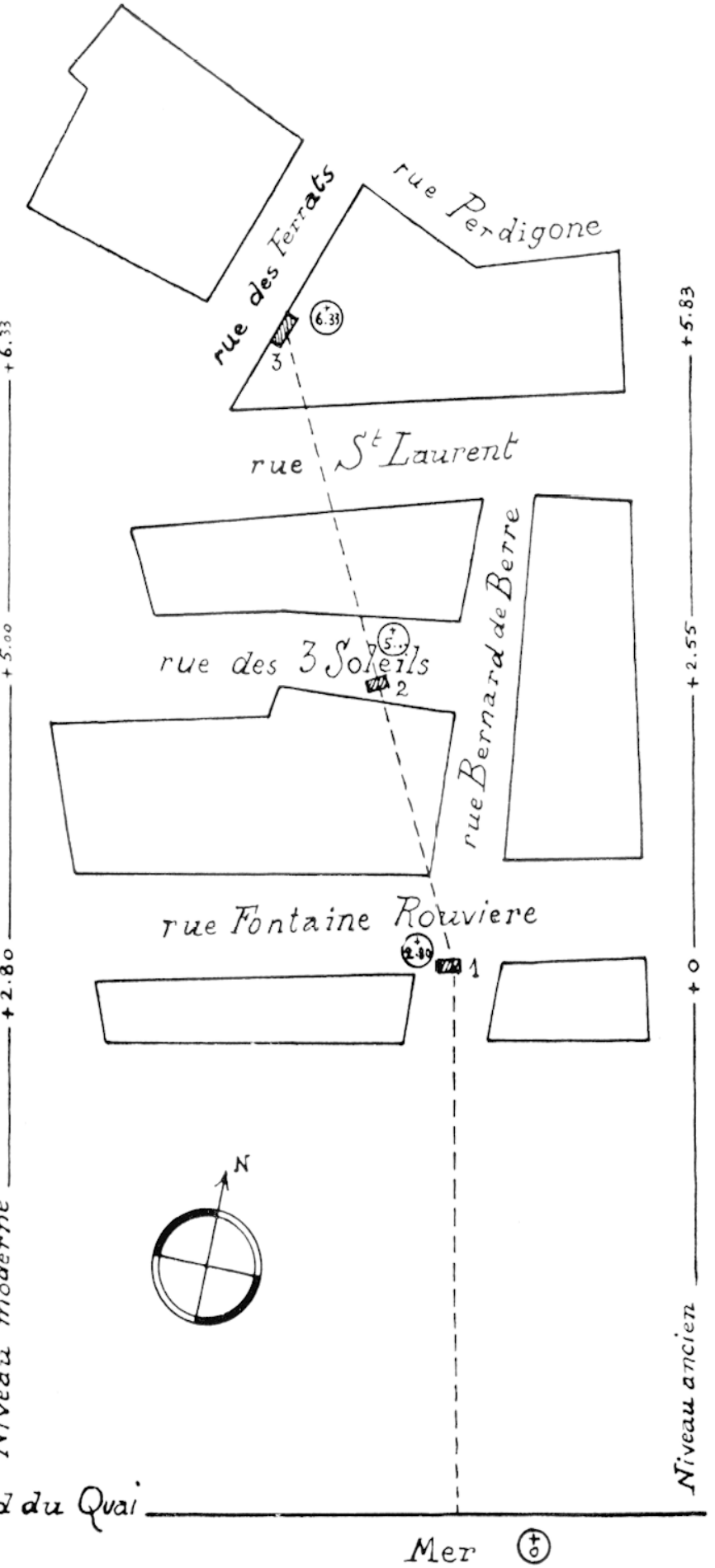

Firg. 1. - Emplacement des sundages. 